\title{
DSpace@MIT
}

\author{
MIT Open Access Articles
}

\author{
Alternative Activity Pattern Generation \\ for Stated Preference Surveys
}

The MIT Faculty has made this article openly available. Please share how this access benefits you. Your story matters.

Citation: He, He et al. "Alternative Activity Pattern Generation for Stated Preference Surveys" Transportation Research Record, vol. 2672, no. 47, 2018, pp. 135-145 (c) 2018 The Author(s)

As Published: https://dx.doi.org/10.1177/0361198118782760

Publisher: SAGE Publications

Persistent URL: https://hdl.handle.net/1721.1/125659

Version: Author's final manuscript: final author's manuscript post peer review, without publisher's formatting or copy editing

Terms of use: Creative Commons Attribution-Noncommercial-Share Alike 


\section{ALTERNATIVE ACTIVITY PATTERN GENERATION FOR STATED PREFERENCE SURVEYS}

\section{He He}

Massachusetts Institute of Technology

77 Massachusetts Avenue, Room 9-549, Cambridge, MA 02139

Tel: 617-955-3922; Email: thehe@mit.edu

\section{Bilge Atasoy}

Massachusetts Institute of Technology

77 Massachusetts Avenue, Room 1-180, Cambridge, MA 02139

Tel: 617-324-7197; Email: batasoy@mit.edu

\section{J. Cressica Brazier}

Massachusetts Institute of Technology

77 Massachusetts Avenue, Room 9-549, Cambridge, MA 02139

Tel: 510-502-8032; Email: cressica@mit.edu

\section{P. Christopher Zegras}

Massachusetts Institute of Technology

77 Massachusetts Avenue, Room 10-403, Cambridge, MA 02139

Tel: 617-452-2433; Email: czegras@mit.edu

\section{Moshe Ben-Akiva}

Massachusetts Institute of Technology

77 Massachusetts Avenue, Room 1-181, Cambridge, MA 02139

Tel: 617-253-5324; Email: mba@mit.edu

Word count: 6250 words text +5 tables/figures $x 250$ words (each) $=7500$ words 


\section{ABSTRACT}

2 We present a systematic method for generating activity-driven, multi-day alternative activity

3 patterns that form choice sets for stated preference surveys. An activity pattern consists of

4 information about an individual's activity agenda, travel modes between activity episodes, and

5 the location and duration of each episode. The proposed method adjusts an individual's observed

6 activity pattern using a hill-climbing algorithm, an iterative algorithm that finds local optima, to

7 search for the best response to hypothetical system changes. The multi-day approach allows for

8 flexibility to reschedule activities on different days and thus presents a more complete view of

9 demand for activity participation, as these demands are rarely confined to a single day in reality.

10 As a proof-of-concept, we apply the method to a multi-day activity-travel survey in Singapore

11 and consider the hypothetical implementation of an on-demand AV service. The demonstration

12 shows promising results with the algorithm exhibiting overall desirable behavior with reasonable

13 responses. In addition to representing the individual's direct response, the use of observed

14 patterns also reveals the propagation of impacts, i.e. indirect effects, across the multi-day activity 15 pattern.

Keywords: Activity Patterns, Activity Rescheduling, Stated Preference Survey, Choice Set Generation. 


\section{INTRODUCTION}

Individuals generally do not make decisions to travel in isolation. Rather, they make these choices simultaneously with, or as a result of, decisions to participate in activities. Accounting for the motivations underlying individuals' activity-travel behavior has become increasingly recognized as essential to understanding today's dynamic urban systems. This activity-based lens is particularly useful for examining complex behavior, such as trip chaining, discretionary travel, as well as the impacts of the range of "soft" measures associated with mobility management. Considering the growing popularity of flexible work hours and shared on-demand modes, and the potentially disruptive changes associated with autonomous vehicles (AVs) and mobility as a service, we need better ways to understand how such innovations will influence individuals' demand for activities and, ultimately, the performance of the mobility and broader urban system.

To understand the possible implications of new products and services "unfamiliar" to a consumer, researchers have long used the stated preference (SP) survey. Travel behavior analysis often uses SP surveys to, say, understand how users might respond to a new mode, such as high speed rail, or infrastructures, such as bike lanes. Such analyses, however, tend to be trip-focused - conditional on a trip being made. New mobility innovations might, however, impact individuals' activity implementation plans or schedules by, for example, allowing more time to partake in discretionary activities in a new location. Thus, understanding the process by which individuals and households schedule their activities remains a core challenge in inferring responses to new mobility or activity options. Even though activity scheduling is something that nearly everyone actually does, to some degree, on a daily basis, formalizing and generalizing it analytically is difficult. Activity scheduling is a multidimensional task that requires the decision maker to choose a number of activities to complete, the sequence of these activities, the time, duration, and location of each activity, and, when relevant, the mode of travel and route between activity locations. Even assuming a reasonable discretization of time and space, the resulting number of possible combinations is both computationally demanding and too large for any person to feasibly consider, cognitively. Generating alternative activity patterns, thus, must aim to be computationally efficient, while mimicking people's actual decision-making processes.

In this paper, we propose a systematic method for generating alternative activity patterns that will form choice sets for stated preference surveys within an activity-based modeling framework. The method takes advantage of detailed multi-day activity diaries collected using Future Mobility Sensing (FMS), a smartphone-based activity-travel survey platform (1). We start with individuals' revealed activity-travel patterns as the base for the new activity patterns. Then, using the proposed method, we adjust the patterns in response to hypothetical scenarios. In addition to representing the individual's direct response, the use of observed patterns also reveals the propagation of impacts across a multi-day activity pattern. Here, we review existing work addressing the challenges of generating alternative activity patterns. We then present the new method and the issues we considered in its development, and finally we show the results of a proof-of-concept application of the proposed method using an activity-travel survey conducted in Singapore.

\section{BACKGROUND}

Since the inception of the activity-based approach to travel demand modeling, researchers have proposed and operationalized numerous activity-based frameworks. Each has taken on the challenge of activity scheduling. Early work established that proposed activity-based models must recognize and take into account the decision-making behavior of individuals and

47 households (2,3). Furthermore, they must be complete in scope, i.e. have sufficiently high 
resolution and flexibility to capture the behavioral changes of interest. Lastly, they must be feasible and practical to operationalize. Early frameworks applied mathematical programming and neural networks in attempts to manage the complexity of the problem $(4,5)$. However, these methods did not simulate how people actually plan their schedules. More recent modelling frameworks have used more sophisticated ways of representing planning and decision-making processes. These approaches have generally fallen on a spectrum between entirely econometricsbased methods, building on discrete choice theory, e.g. SimMobility (6), to largely rule-based methods that build on decision heuristics, e.g. TASHA $(7,8)$ and ALBATROSS (9). Most modelling frameworks can be considered hybrids, applying a combination of heuristics and econometrics or at least using the concept of utility-maximization to simulate rationality. Pinjari and Bhat provide a comprehensive overview of activity-based modelling frameworks (10).

A major challenge to operationalizing activity-based models has to do with realistically representing how individuals schedule activities and, thus, what alternative activity patterns might exist for a given individual. For the purposes of an activity-based SP survey, aimed at understanding how an individual's mode and activity preferences might change in the face of new alternatives, realism would be gained by presenting activity pattern alternatives based on respondents' observed activity patterns. This requires methods that can capitalize on the availability of existing patterns and estimate the utility of activities for a given individual. The AMOS model, a micro-simulator designed to predict behavioral responses to travel demand management (TDM) measures, offers a precedent (5). AMOS uses a neural network approach to generate TDM options based on revealed preference and SP survey data. Its focus, however, is on commuters and it uses pre-coded response types. For example, in its application to the Washington DC area, seven response types are possible, including "no change”, "change work departure time”, “work from home”, and four different options for work trip mode changes (11). The neural network calculates probabilities for each response type based on socio-economic characteristics and existing activity patterns, and responses are selected using Monte Carlo simulation. Afterwards, the model adjusts the rest of the activity pattern to accommodate the response if necessary. However, these adjustments are mostly constraint-based. Furthermore, the prescriptive nature of the pre-coded responses do not capture the diversity of people's potential response strategies (12) and largely eliminate the possibility of response discovery.

As micro-simulators have become more advanced, and as technology-based survey methods have allowed for more comprehensive time-use and travel data collection, e.g. CHASE (13), REACT! (14), and UTRACS (15), several modelling frameworks have incorporated nearterm rescheduling modules. These frameworks adapt agents’ planned activity patterns in response to unforeseen circumstances that agents do not take into consideration in the original activity scheduling phase. The components of this problem, i.e. an existing activity pattern and a change in circumstances triggering adaptations, are the same as those we are faced with in generating alternative activity patterns for stated preference surveys. Hence, the methods employed by various activity-based microsimulation frameworks for finding solutions to the rescheduling problem might also apply to our problem. AURORA, a rescheduling module developed as an extension to the ALBATROSS framework, assigns utility to activities based on their duration $(16,17)$. Specifically, the utility function is S-shaped, monotonically increasing with activity duration, and has horizontal asymptotes corresponding to the minimum and maximum utilities that can be derived from an activity. The second derivative of the utility function is positive until it reaches an inflection point after which it becomes negative. AURORA allows for a diverse combination of responses, including changes to travel mode, episode durations, locations, and sequence, as well as insertion and deletion of episodes. The model 
chooses a response using an iterative optimization procedure that imitates bounded rationality and accounts for people's resistance to change by introducing a disutility of each change operation. The primary drawbacks to the AURORA model are the difficulty of estimating the large number of parameters of the S-shaped utility function $(18,19)$ and its lack of multi-day schedule integration (20). Another agent-based modeling framework, MATSim, uses a simpler two-parameter utility formulation, in which utility is a logarithmic function of activity duration (21). In other words, utility is monotonically increasing with activity duration with an everdiminishing marginal effect. A rescheduling extension for MATSim now applies an iterative approach similar to that in AURORA (22). However, this extension simplifies the procedure considerably in order to reduce computational demand.

\section{ACTIVITY PATTERN GENERATION PROCEDURE}

We propose a procedure for generating personalized alternative activity patterns triggered by changes in the transportation system or in the characteristics of activities. The procedure builds on the concepts and methods developed in AURORA (17), the MATSim rescheduling module (22), and others. Specifically, our algorithm is also an iterative optimization procedure that uses elementary operations to incrementally search for better alternatives. We assume the logarithmic formulation for activity utility and make use of the same elementary operations as implemented in MATSim, and we also adopt a notion of resistance to change similar to AURORA, however we apply it directly to changes to schedule times rather than the number of elementary operations. Despite the similarities, our objective differs slightly. We are not necessarily interested in the most likely response to system change; rather, we want to explore feasible responses by generating alternative activity patterns that account for activity demands. Furthermore, we examine multi-day activity patterns and allow for flexibility to schedule activities on different days, e.g. a disruption on a given day might result in the rescheduling of a subsequent activity to a different day in the multi-day pattern. This approach presents a more complete view of an individual's activity demand, which is rarely confined to a single day in reality. Lastly, the application of the methods to comparatively small samples in stated preference surveys, as opposed to a synthesized population of agents, somewhat relaxes the constraints on computation time.

For our proposed procedure, an alternative activity pattern is generated using an iterative hill-climbing algorithm to search for a local maximum utility, given a set of preference parameters. The hill-climbing algorithm is a mathematical optimization technique that takes an observed pattern and incrementally improves it by changing one element at a time. The algorithm is complete when the utility of the pattern can no longer improve by changing any one element. Some modellers question the appropriateness of utility maximization from a behavioral standpoint. However, its application here is local in nature: the number of possible activity patterns is severely limited by the procedure of changing single elements combined with the requirement that each iteration of the algorithm must score better than the last, i.e. 'move up the hill'. In other words, alternative patterns cannot change drastically by 'jumping between hills'. The hill-climbing algorithm can be interpreted as a search for the best pattern that is similar to the observed pattern. The sequential approach, together with the non-exhaustive search procedure, considerably reduces computation time compared to simultaneous optimization approaches. This is especially the case as the activity patterns become more complex, i.e., with the introduction of more activity types, locations, modes, etc. The procedure generates each alternative activity pattern as the 'optimal choice' for a given set of preference parameters; thus, we can generate a different alternative pattern by assuming a different set of parameters. In other 
words, a complete choice set of alternative activity patterns can be generated by varying the individual's assumed preferences, either systematically or randomly. The preference parameters describe an individual's activity demands, modal preferences, value of time, and flexibility.

Figure 1 shows the steps within each iteration of the algorithm. For the first iteration, the observed activity pattern serves as the baseline. The final activity pattern output from the procedure is the alternative activity pattern. During each iteration of the hill-climbing algorithm, we generate and propose numerous activity patterns, called proposed activity patterns. Lastly, within these proposed activity patterns, an activity agenda consists of a set of consecutive episodes, e.g. home-work-home-shop-home. This activity agenda does not provide information about the timing, duration, or location of episodes, or any information about travel.

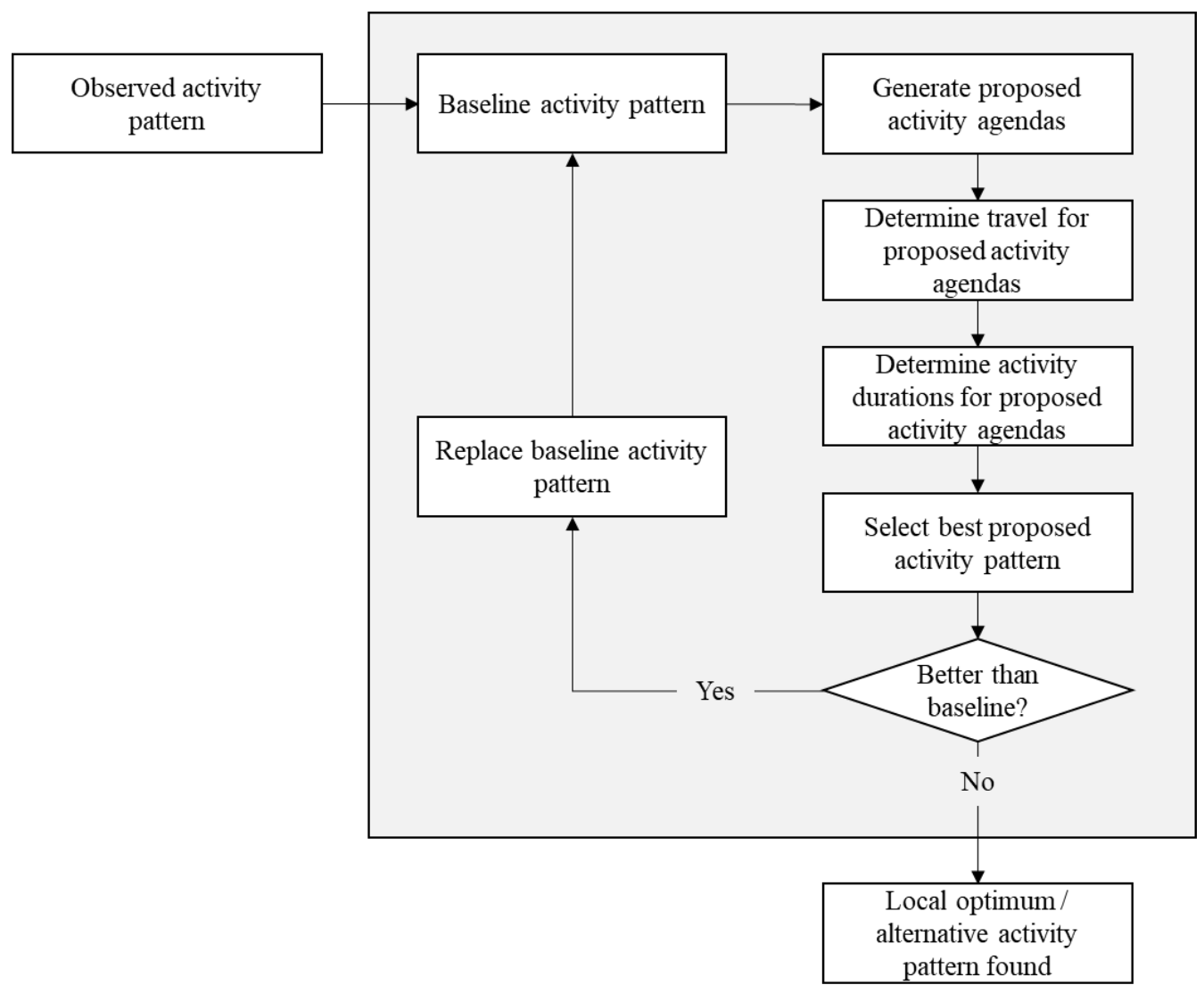

FIGURE 1 Alternative activity pattern generation procedure.

\section{Generate proposed activity agendas}

Within each iteration, the agenda of the baseline pattern serves as a skeleton. We generate every unique proposed agenda that we can possibly create by applying the single elementary operations to the baseline agendas. The number of proposed agendas generated in this step depends primarily on the number of episodes in the observed activity pattern and the number of different activity types. Figure 2 illustrates the elementary operations used to modify the baseline agenda. These are the same elementary operations used by (22).

First, an attempt is made to insert a new episode between two episodes in the baseline agenda (Figure 2a). This operation occurs with every activity type between every pair of consecutive episodes in the baseline agenda. To preserve the "home" episodes as the anchors of the daily agendas, new episodes are not inserted at the beginning or end of the agenda. If the 
inserted episode is different in type from both the preceding and subsequent adjacent episodes in the agenda, a new agenda is created. Otherwise, the procedure continues on to the next potential slot or activity type.

Next, an attempt is made to remove a single episode at a time (Figure 2b), for every episode in the baseline agenda. If the episode is not an overnight "home" episode, it is removed and a new agenda is created. Otherwise, the procedure moves on to the next episode in the agenda. Following the episode removal, if two consecutive episodes are of the same type, one of them is removed.

Finally, two episodes in the baseline agenda are swapped if neither is an overnight "home" episode (Figure 2c). This operation is attempted for every possible episode pair combination in the agenda. By allowing this swapping operation, proposed activity patterns with higher total utility can be generated. However, these patterns will likely also bear less resemblance to the individual's observed activity pattern. Whether or not swapping generates reasonable changes in general requires further testing. For our example application in the next section, we do not apply the swapping operation.

a. INSERT

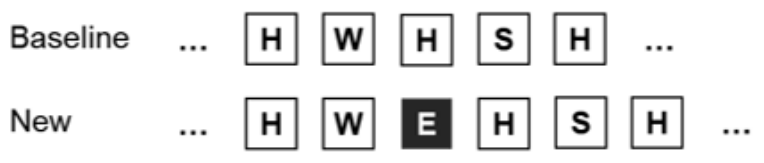

b. REMOVE

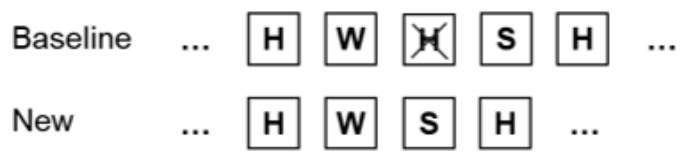

c. SWAP

FIGURE 2 Example of the INSERT, REMOVE, and SWAP operations.

\section{Determine travel for proposed activity agendas}

This step determines the mode for every trip in every agenda using the principle of utility maximization. First, each proposed agenda is aligned with the observed agenda and the closest episodes of the same type within the same day are paired across the two agendas. Note that this comparison is with the original, observed agenda, which is generally different from the baseline agenda after the first iteration. Typically, episodes that have undergone the "insert" or "remove" operations cannot be paired, because they do not have a corresponding episode in the observed pattern or proposed agendas, respectively. For paired episodes, the activity location remains the same. For unpaired episodes with multiple possible activity locations (e.g. if the user could complete a shopping activity at similar stores in different locations), we take a myopic approach. Specifically, trips are evaluated sequentially and the mode choice becomes a joint modedestination choice, because each trip entails the choice between all possible modes to all possible 
might have adverse effects on downstream mode-destination choices; however, we do not account for these effects at present. A more nuanced method for selecting activity locations is part of the ongoing work.

For a given origin-destination pair and mode $m$, we assume that the utility of a trip $V_{\text {trip }}$ is a function of the travel time and travel cost:

$$
V_{\text {trip }}=\beta_{m}+\beta_{m, T} \cdot T_{m}+\beta_{m, C} \cdot C_{m}
$$

where:

- $\quad \beta_{m}$ is an alternative specific constant for mode $m$;

- $\quad \beta_{m, T}$ is the parameter for travel time by mode $m$;

- $T_{m}$ is the travel time by mode $m$;

- $\quad \beta_{m, C}$ is the parameter for travel cost by mode $m$; and

- $C_{m}$ is the travel cost by mode $m$.

The parameters can be varied for this utility function to generate a set of different activity patterns, or the parameters can be estimated from a pre-survey. In addition to the utility of the trip itself, the selection of mode should account for the opportunity cost of travel time, i.e., time that could be spent productively on other activities. For example, if it takes a person 20 minutes to drive home and 60 minutes to walk home from work, the drive option will provide him with an additional 40 minutes to spend at home or to carry out another activity later on, from which he can derive additional utility. In other words, the duration of the trip affects utility outside of the trip itself. However, the selection of modes should account for this opportunity cost. Therefore, we estimate the magnitude of the opportunity cost for a trip $O C$ as:

$$
O C=-\frac{V_{\text {activity,total }}}{D} \cdot T_{m}
$$

where:

- $V_{\text {activity,total }}$ is the total utility from all episodes in the observed activity pattern; and

- $\quad D$ is the total duration spent on activities in the observed activity pattern.

The procedure of assigning utility to activities follows in the next section. For each trip, the selected mode and destination, if applicable, are that which yield the maximum total utility, i.e. the minimum sum of trip disutility and opportunity cost of travel time.

\section{Determine activity durations for proposed activity agendas}

With travel determined, we now determine the duration of each episode in each activity agenda such that the total utility of each agenda is maximized. We assume that the utility of an activity $a$ is a function of the duration of the activity $D_{a}$ :

$$
V_{\text {activity }}=\beta_{a} \cdot \ln \left(\alpha_{a} \cdot D_{a}\right)
$$

Here $\beta_{a}$ and $\alpha_{a}$ are scale and shape parameters, respectively, for the utility of activity type $a$. This formulation of utility follows the method that Charypar and Nagel applied to MATSim (20). Again, we can derive or estimate the parameters such that the observed activity pattern is optimal without system changes, or we can vary them to generate a set of different activity patterns. For 
positive parameters, the utility function is monotonically increasing with duration but diminishing at the margin. The diminishing marginal effect can be interpreted as fatigue, boredom, or other forms of efficiency loss as the duration of an activity increases. For some activities in which utility is not necessarily derived intrinsically, such as work or household chores, equation (3) models the extrinsic value of the activity, such as a salary or a clean house. In this framework, the scheduling process is activity-driven, because travel only occurs when the utility derived from an activity outweighs the disutility of travelling there. Furthermore, if we use reasonable values for the $\beta_{a}$ and $\alpha_{a}$ parameters, the principle of utility-maximization ensures a balanced composition of activities, since the diminishing marginal utility, i.e. boredom or fatigue, encourages individuals switch to activities that are more pressing or rewarding. We should also note that because the utility function (3) is non-linear, calculating utility based on duration per episode versus per day or per week will yield different results (Figure 3). Here, three short episodes, each with duration $d_{a}$, yield a total 41.7 utility units if calculated per episode. However, the same three episodes yield only 24.8 utility units if the episode durations accumulate over the analysis period.

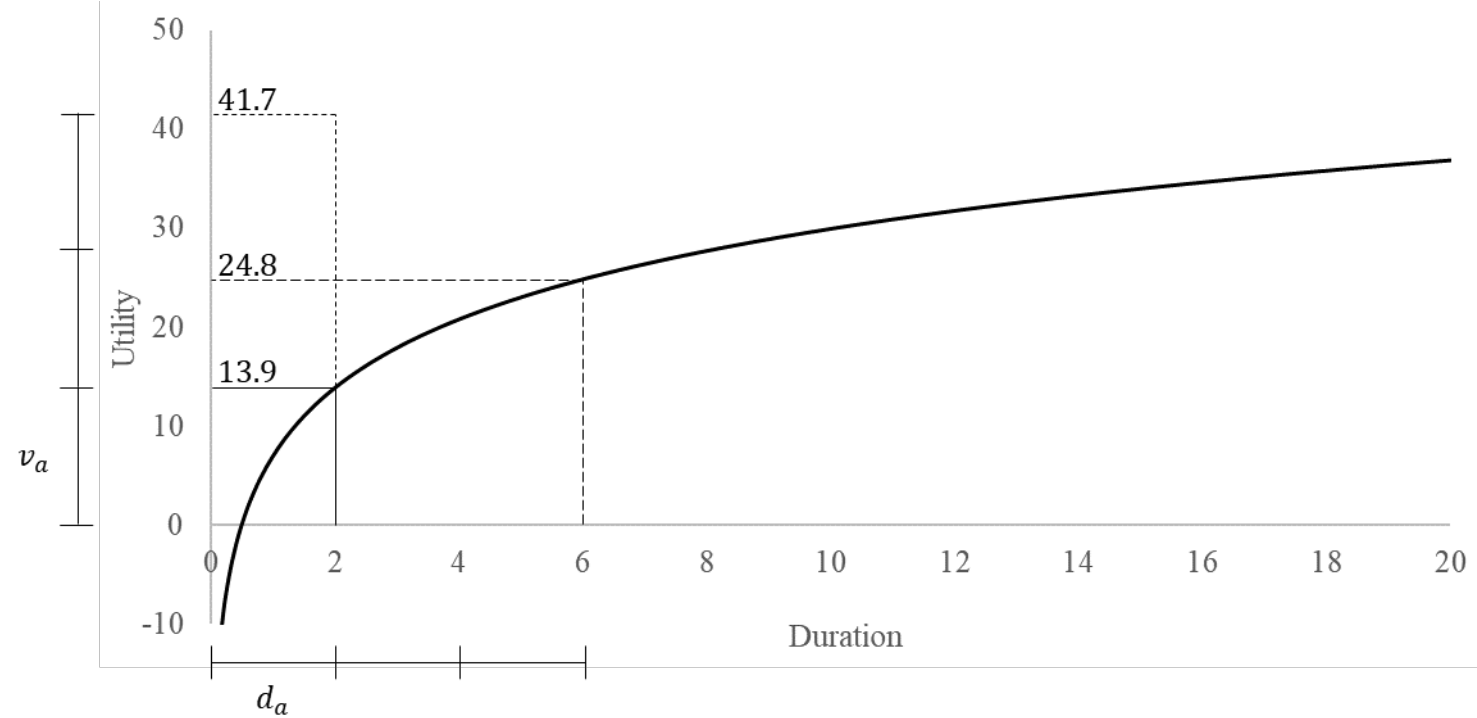

FIGURE 3 Illustration of activity utility discrepancy for different utility accumulation methods.

Calculating utility on a per episode basis generally results in more frequent short episodes, because the marginal utility is higher for short episodes. On the other hand, calculating utility on a per week basis results in fewer long episodes in order to minimize the disutility of travel. We handle this discrepancy by distinguishing between mandatory/maintenance activities and discretionary activities. In particular, we accumulate activity durations over the multi-day analysis period for mandatory and maintenance activities, such as work, shop, and personal errands, while we use daily accumulation for discretionary activities, such as social and recreational.

Beyond the utility of the activity itself, proposed episodes are penalized for changing the individual's schedule, i.e. the observed activity pattern. In principle, this is similar to the disutility of change used by (17). However Joh et al. apply the change to the operation itself, whereas our disutility of change is a function of differences in episode start times between episodes in the observed pattern and the new pattern. Only proposed episodes that successfully 


6

7

pair with episodes in the observed agenda will produce this disutility of change. In other words, newly inserted episodes and episodes that shifted to different days do not have an associated disutility of change. For an episode of activity type $a$, equation (4) gives the disutility of change, $V_{\text {change: }}$

$$
V_{\text {change }}=-\frac{i_{a}}{f} \cdot\left(t_{\text {start }, \text { new }}-t_{\text {start }, \text { obs }}\right)^{2}
$$

where:

- $i_{a}$ is a parameter representing the inertia of activity type $a$;

- $f$ is the flexibility of the individual;

- $t_{\text {start,new }}$ is the start time of the episode in the proposed pattern; and

- $t_{\text {start,obs }}$ is the start time of the episode in the observed activity pattern.

Intuitively, activity types that are more difficult to move temporally have higher inertia and produce greater penalties when their start times have to change. On the other hand, flexible individuals experience lower penalties for changes overall. Similar to other parameters in this framework, the activity-specific inertia and flexibility parameters can be varied to generate different activity pattern alternatives, or they can be derived from a pre-survey or a participant's observed pattern. As part of our ongoing work, we are testing different functional forms; the current formulation is only an a priori hypothesis of how we penalize change. However, validating these formulations as well as the parameters is challenging, and the methods to do so empirically are not obvious.

With all travel already fixed, the duration of each episode can be determined by maximizing the utility of the proposed pattern. The optimization problem is formulated as:

(1)

\section{where:}

- $\quad d_{e}$ is the duration of an episode $e$;

- $\quad D_{a}$ is the sum of durations of episodes $e$ of activity type $a$ that accumulate by day or by analysis period;

- $\quad A$ is the set of all activity types $a$;

- $\quad E$ is the set of episodes $e$ within the day being optimized;

- $\quad E^{\prime}$ is the set of episodes $e^{\prime}$ that pair with episodes in the observed pattern within the day being optimized, i.e. a subset of $E$;

- $\quad R$ is the set of all trips $r$ within the day being optimized;

- $\quad n e w$ in the subscript denotes that the value is from the new pattern;

- $\quad$ obs in the subscript denotes that the value is from the observed pattern; and

- $\quad t_{\text {start,new,e }}$ is the start time of episode $e$ in the new activity pattern, i.e.

$t_{\text {start,new }, e}=0$ for $e=0$, and $t_{\text {start,new,e }}=t_{\text {start,new,e-1 }}+d_{e-1}$ for $e>0$. 
Because utility for non-discretionary activities accumulates beyond a single day, the episode durations within the day depend on episode durations on other days within the analysis period, as these episode durations undergo optimization. This procedure can be interpreted as the individual's knowledge of the past and plan for the future. The individual currently bases his plan for the future on his observed pattern. Consider, for example, work durations over a week. On Monday a person knows how much he should work that week (reflected by the $\beta$-parameter for work). He also has a plan for how much he will work on each day and will try to work a certain number of hours on Monday, so that the total hours add up to the planned hours. However, if he inserts an additional activity on Monday, he will work fewer hours on Monday than in the observed pattern. Subsequently, on Tuesday, he will know he worked too little the previous day and will try to compensate, so that the total hours once again add up.

\section{Select best alternative activity pattern}

Finally, the total utility of each proposed activity pattern is evaluated:

$$
V_{\text {total }}=\sum V_{\text {trip }}+\sum V_{\text {activity }}+\sum V_{\text {change }}
$$

Note that we do not include the opportunity cost of travel time here, because it is already accounted for in $V_{\text {activity }}$.

If the best of the proposed activity patterns has a higher utility than the baseline pattern, another iteration of the algorithm runs using the agenda of the best proposed pattern as the new baseline. Otherwise, the current baseline is selected as the best pattern for the given input parameters.

\section{PROOF-OF-CONCEPT APPLICATIONS}

We tested this proposed procedure for alternative activity pattern generation on a dataset from a multi-day activity survey conducted in Singapore in 2013 (1). This smartphone-based survey contains detailed travel, activity, and location information from consecutive weekdays, e.g. Monday through Friday in the same week. Figure 4a shows one participant's observed weekly activity pattern. In a complete SP experiment, we would collect multi-day or multi-week activity diaries for each respondent to establish a baseline. The respondent would also complete a presurvey, which along with the observed activity patterns inform his preference parameters. We would then change system variables to reflect a hypothetical scenario, e.g. increased/decreased travel times by certain modes, restricted mode choices, etc. Then using the proposed method and different sets of input parameter, we generate different alternative activity patterns that form a choice set. In general, input parameters can be derived from the observed patterns or the respondent's pre-survey. However, for parameters that will be used to estimate a model, the input parameters must be generated randomly to avoid endogeneity.

Here, we consider a hypothetical scenario in which on-demand AV service, Automated Mobility On-Demand (AMOD), is introduced, and examine examples of alternative activity patterns generated for the abovementioned survey participant. Given that such a service does not yet exist, it is difficult to judge the method's predictive accuracy. However, our objective is only to generate numerous feasible alternatives that form a choice set for respondents to choose between, thus perfect predictions of actual responses are not necessary at this stage. For the purposes of this proof-of-concept demonstration, we assume that AMOD travel times are the same as travel times by automobile at twice the cost of completing the same trip by transit. Depending on the individual's preferences, adopting AMOD for one or more trips might improve 
the overall utility of their activity pattern. We select the preference parameters for this demonstration (Table 1 ) by assuming that the mode-specific constants, $\beta_{m}$, are 0 for all modes and that other preference parameters associated with travel and change fall within a reasonable range. These a priori values were assumed for proof-of-concept demonstration purposes. A different set of parameters would generate different alternative patterns. Lastly, we derive the preference parameters associated with activities from the observed activity pattern, such that activity choices would remain similar if there were no changes to the system.

TABLE 1 Example user parameters

\begin{tabular}{|c|c|c|}
\hline Parameter Type & Value & Value \\
\hline Travel Modes & $\beta_{m, T}[u t i l i t y / \min ]$ & $\beta_{m, C}[u t i l i t y / \$]$ \\
\hline Walk & -5.5 & -4.0 \\
\hline Car & -1.8 & -4.0 \\
\hline Transit & -1.2 & -4.0 \\
\hline AMOD & -0.8 & -4.0 \\
\hline Activities & $\alpha_{a}[1 /$ hour $]$ & $\beta_{a}$ [utility] \\
\hline Home & 1.0 & 12.0 \\
\hline Work & 0.3 & 47.8 \\
\hline Shop & 102.1 & 0.1 \\
\hline Personal & 9.1 & 1.3 \\
\hline Meal & 9.1 & 1.3 \\
\hline \multicolumn{3}{|l|}{ Change } \\
\hline$f$ & 150 [no unit] & \\
\hline$i_{\text {home }}$ & 10 [utility / hour'] & \\
\hline$i_{\text {work }}$ & 200 [utility / hour ${ }^{2}$ ] & \\
\hline$i_{\text {shop }}$ & 20 [utility / hour ${ }^{2}$ ] & \\
\hline$i_{\text {personal }}$ & 50 [utility / hour ${ }^{2}$ ] & \\
\hline$i_{\text {meal }}$ & 20 [utility / hour ${ }^{2}$ ] & \\
\hline
\end{tabular}

Work, shopping, and personal errands fall into the category of mandatory/maintenance activities, and thus their durations accumulate over the entire survey period for utility calculation purposes. We categorize home and meal breaks as discretionary activities. Arguably, home can be considered a mandatory/maintenance activity; however, because we never remove overnight home episodes, the remaining home-related decisions are of a discretionary nature, e.g. how long to spend at home, whether to return home between activities, or whether to extend the tour.

Figure $4 \mathrm{~b}$ presents the generated alternative activity pattern. In this pattern, the survey participant uses AMOD for two trips, once Thursday night on his way home from the personal errand and once Friday evening for his shopping trip. The convenience of AMOD also introduced a new home episode on Friday, making shopping a separate home-based tour. However, as expected, the relatively high cost of the service results in limited adoption and minimal impact on the observed activity pattern, with the assumed preference parameters.

Figure 4c presents a different final alternative activity pattern, in which we explore the impact of complete AMOD adoption. We removed the walk, car, and transit mode options and halved the magnitude of the AMOD parameters, i.e. $\beta_{A M O D, T}=-0.4 / \mathrm{min}$ and $\beta_{A M O D, C}=-2 / \$$. The shorter AMOD travel times allow the survey participant to leave home 
1 later in the morning and return home earlier in the evening. For example, on Thursday he would 2 arrive home at 8:26 pm as opposed to 8:55 pm. At the same time, he would add a meal episode 3 on Tuesday, and available work time increases from 47.8 hours to 48.6 hours.

$4 \quad$ These examples also highlight the limitations of the proposed method in its current state. 5 The sequential, iterative approach does not account for travel times that vary according to the 6 time of day; thus, we base all travel times in the alternative patterns on evening peak travel 7 conditions. Not only does this potentially affect the selection of mode, it also inaccurately 8 determines the time available for activities. Similarly, activity demands are constant throughout 9 the day, a potentially unrealistic property that the additional hour-long meal break before 8 am on 10 Tuesday in the second alternative pattern illustrates. 


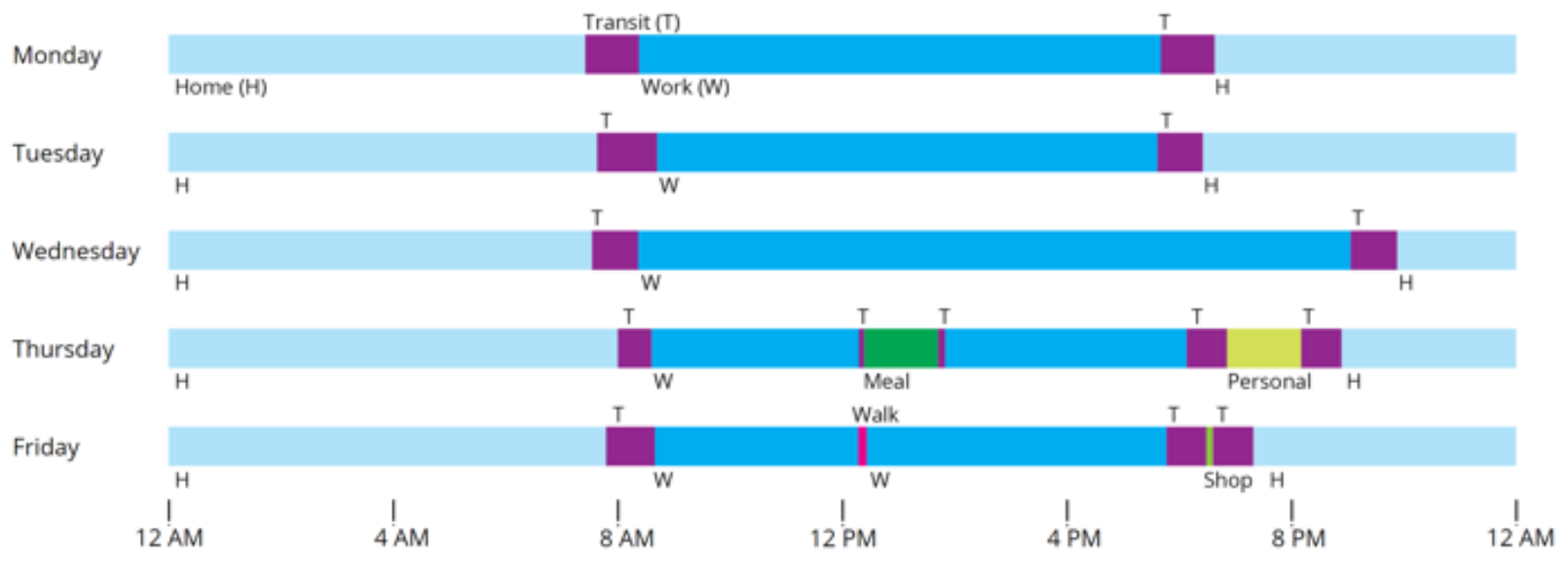

(a) Original pattern

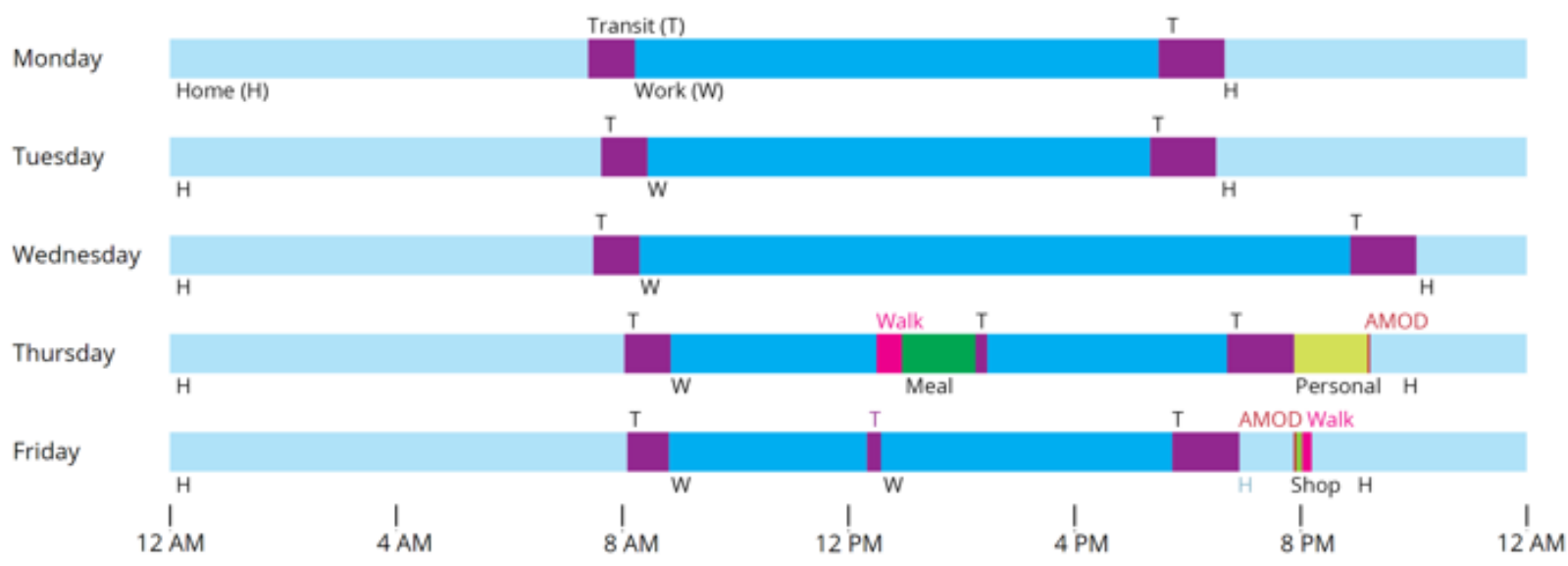

(b) Partial AMOD adoption

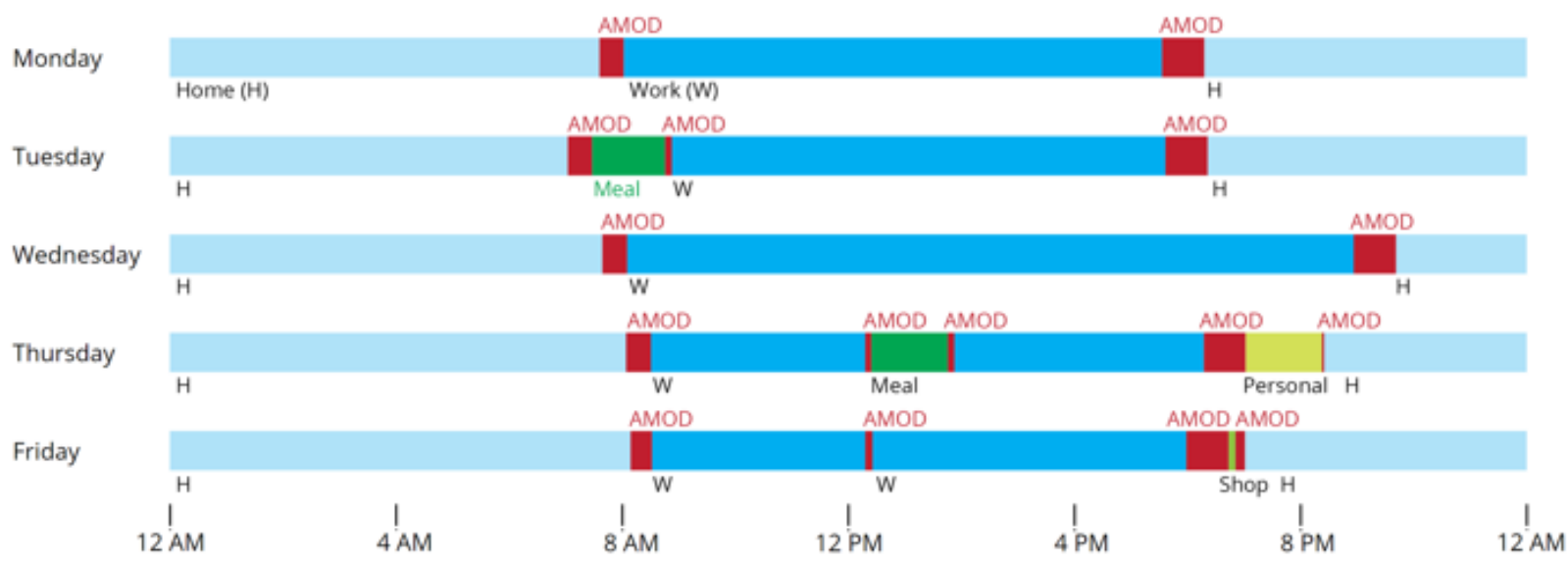

(c) Complete AMOD adoption

FIGURE 4 An individual's observed weekly activity pattern (a), example alternative activity pattern with partial AMOD adoption (b) and complete AMOD adoption (c). 


\section{CONCLUSION AND FUTURE DIRECTION}

2 We have presented a new method for generating activity-driven, multi-day alternative activity 3 patterns, designed for use in stated preference surveys. This method generates alternatives using a local optimization algorithm that searches for the best response to a system change that is similar to the survey participant's observed patterns. The alternative patterns also allow us to explore the propagation of indirect effects resulting from the participant's direct response. As a proof-of-concept, we applied the method to data from a multi-day activity-travel survey in Singapore and considered the hypothetical implementation of an on-demand AV service. The demonstration showed promising results with the algorithm exhibiting overall desirable behavior with reasonable responses. However, the examples highlight the method's current limitations, namely inflexibility vis-à-vis activity demand and travel times depending on the time of day. In our continued work, we plan to address these limitations as well as to allow for substitution of activities within activity types, e.g. the substitution of office work with teleworking. Incorporating additional ways to constrain alternatives to the observed pattern might also enable more nuance in the generated activity patterns. Currently the measure of change is considered with the difference in start times of activities but the change in the total number of activities is another candidate to impose further constraints.

As we improve the method, we will test the generated activity patterns on respondents to calibrate and ensure that we are generating relevant alternatives. We will soon employ the proposed method in two activity-based experiments focusing on AMOD adoption and carbon emissions feedback. For the first experiment, a stated preference survey, we will test how to align the experimental treatments, namely the AMOD services, with the activity generation procedure we proposed. For instance, instead of presenting the participants with the full range of AMOD adoption possibilities shown in the proof-of-concept above, we can first gather the participants' stated preferences for their level of adoption of AMOD services or subscription programs. Then, we can use their adoption preferences to tailor the set of alternative activity patterns that we present to the participants in the second part of the stated preference survey and evaluate whether the participants accept the impact of the AMOD treatment on their overall activity patterns. The proposed method can also be developed into a travel-activity planning advisory application that automatically generates feedback and personalized advice based on users' stated or revealed preferences. In the second experiment, we will test the effectiveness of smartphone-based activity-travel and carbon emissions feedback delivery for encouraging sustainable behavior change. The algorithm's multi-day pattern generation capability can support recommendations for blended travel mode choices over extended study periods. Additionally, by accounting for the multidimensional impacts of changing activities and travel in realistic alternative patterns, this method can help test the effectiveness of an assisted behavioral planning program.

\section{ACKNOWLEDGEMENTS}

The MIT Energy Initiative (MITEI) Seed Grant Fund and the National Research Foundation Singapore, through the FM IRG research group at the Singapore-MIT Alliance for Research and Technology, supported this research. 


\section{REFERENCES}

1. Cottrill, C., F. Pereira, F. Zhao, I. Dias, H. B. Lim, M. Ben-Akiva, and C. Zegras, Future Mobility Survey: Experience in Developing a Smart-Phone-Based Travel Survey in Singapore, Transportation Research Record: Journal of the Transportation Research Board, issue 2354, pp. 59-67, 2013.

2. Bowman, John L., and Moshe Ben-Akiva. Activity based travel forecasting, Activity based travel forecasting conference, New Orleans, June 1996.

3. Axhausen, Kay W., and Tommy Gärling. Activity-based Approaches to Travel Analysis: Conceptual Frameworks, Models, and Research Problems. Transport Reviews, 12, 1992, pp. 323-341, https://doi.org/10.1080/01441649208716826

4. Recker, Wilfred W. The household activity pattern problem: General formulation and solution. Transportation Research Part B: Methodological, Volume 29, Issue 1, 1995, pp. 61-77, http://dx.doi.org/10.1016/0191-2615(94)00023-S.

5. Kitamura, Ryuichi, and Satoshi Fujii. Two Computational Process Models of Activity-Travel Behavior. Theoretical Foundations of Travel Choice Modeling, 1998, pp. 251-279

6. Lu, Yang, Muhammad Adnan, Kakali Basak, Francisco Câmara Pereira, Carlos Carrion, Vahid Hamishagi Saber, Harish Loganathan, and Moshe E. Ben-Akiva. SimMobility mid-term simulator: A state of the art integrated agent based demand and supply model. Transportation Research Board, Washington DC, 2015.

7. Miller, Eric, and Matthew Roorda. Prototype model of household activity-travel scheduling. Transportation Research Record: Journal of the Transportation Research Board 1831, 2003, pp. 114-121.

8. Roorda, Matthew J., Eric J. Miller, and Khandker M.N. Habib, Validation of TASHA: A 24-H Activity Scheduling Microsimulation Model, Transportation Research Part A: Policy and Practice, Volume 42, Issue 2, 2008, pp. 360-75. doi:10.1016/j.tra.2007.10.004.

9. $\quad$ Arentze, Theo, and Harry Timmermans, Albatross: A Learning Based Transportation Oriented Simulation System, Eirass Eindhoven, 2000.

10. Pinjari, Abdul Rawoof, and Chandra R. Bhat. Activity-Based Travel Demand Analysis, A Handbook of Transport Economics 10, 2011, pp. 213-248.

11. RDC, Activity-based modeling system for travel demand forecasting, Metropolitan Washington Council of Governments, US Department of Transportation, US Environmental Protection Agency, Washington DC, 1995.

12. Doherty, Sean, Martin Lee-Gosselin, Kyle Burns, and Jean Andrey, Household Activity Rescheduling in Response to Automobile Reduction Scenarios, Transportation Research Record: Journal of the Transportation Research Board, no. 1807, 2002, pp. 174-181.

13. Doherty, Sean T., and Eric J. Miller, A Computerized Household Activity Scheduling Survey, Transportation, Volume 27, Issue 1, 2000, pp. 75-97.

14. Lee, Ming S., and Michael G. McNally, On the structure of weekly activity/travel patterns, Transportation Research Part A: Policy and Practice, Volume 37, Issue 10, 2003, pp. 823-839.

15. Auld, Joshua, Martina Z. Frignani, Chad Williams, and Abolfazl Kouros Mohammadian, Results of the UTRACS Internet-Based Prompted Recall GPS Activity-Travel Survey for the Chicago Region, $12^{\text {th }}$ World Conference in Transportation Research, Lisbon, Portugal, 2010. 
16. Timmermans, Harry, Theo Arentze, and Chang-Hyeon Joh, Modeling effects of anticipated time pressure on execution of activity programs, Transportation Research Record: Journal of the Transportation Research Board 1752, 2001, pp. 8-15, doi:10.1016/j.tra.2007.10.004.

17. Joh, Chang-Hyeon, Theo Arentze, and Harry Timmermans, Modeling Individuals’ Activity-Travel Rescheduling Heuristics: Theory and Numerical Experiments, Transportation Research Record: Journal of the Transportation Research Board, no. 1807, 2002, pp. 16-25.

18. Joh, Chang-Hyeon, T. A. Arentze, and H. J. P. Timmermans, Estimating NonLinear Utility Functions of Time Use in the Context of an Activity Schedule Adaptation Model, $10^{\text {th }}$ International Conference on Travel Behavior Research, 2003.

19. Nagel, Kai, Kay W. Axhausen, Benjamin Kickhöfer, and Andreas Horni, Research Avenues, In The Multi-Agent Transport Simulation MATSim (Andreas Horni, Kai Nagel, and Kay W. Axhausen, ed.), Ubiquity Press, 2016, pp. 533-42, doi:10.5334/baw.97.

20. Arentze, T. A., Claudia Pelizaro, and H. J. P. Timmermans. Implementation of a Model of Dynamic Activity-Travel Rescheduling Decisions: An Agent-Based Micro-Simulation Framework. In Proceedings of the Computers in Urban Planning and Urban Management Conference, Volume 48, 2005.

21. Nagel, Kai, Benjamin Kickhöfer, Andreas Horni, and David Charypar, A Closer Look at Scoring. In The Multi-Agent Transport Simulation MATSim (Andreas Horni, Kai Nagel, and Kay W. Axhausen, ed.), Ubiquity Press, 2016, pp. 2334.doi:10.5334/baw.3.

22. Balac, Milos, and Kay W. Axhausen Activity rescheduling within a multi-agent transport simulation framework (MATSim), Transportation Research Board, Washington DC, 2017. 